'Servicio de Medicina Interna, Hospital de Puerto Montt, Puerto Montt, Chile.

${ }^{2}$ Departamento de Medicina. Universidad San Sebastián. Puerto Montt. Chile.

${ }^{3}$ Escuela de Medicina. Universidad San Sebastián. Puerto Montt. Chile.

${ }^{4}$ Laboratorio de Microbiología Hospital de Puerto Montt, Puerto Montt, Chile.

aédico residente de Medicina Interna, Universidad San Sebastián, Hospital de Puerto Montt, Puerto Montt, Chile.

Fuente de apoyo financiero: no hubo.

Recibido el 10 de diciembre de 2013, aceptado el 5 de junio de 2014.

Correspondencia a: Dr. Raúl Riquelme Bellavista 123 oficina 402 , Puerto Montt. rauloriquelme@gmail.com

\section{Infección por virus respiratorios en adultos hospitalizados en un Servicio de Medicina Interna}

\author{
RAÚL RIQUELME ${ }^{1,2}$, MARÍA LUISA RIOSECO ${ }^{3,4}$, \\ YASNA AGÜERO ${ }^{1,3, a}$, DANIELA UBILLA ${ }^{1,3, a}$, PAMELA MECHSNER ${ }^{1,3, a}$, \\ CARLOS INZUNZA ${ }^{1,2}$, MAURICIO RIQUELME ${ }^{1,2}$
}

\section{Respiratory virus infections in adult patients hospitalized in an internal medicine unit}

Background: Respiratory viral infections (RVi) can be associated with a wide range of clinical manifestations. Aim: To investigate the frequency and clinical manifestations of RVi among adult patients during winter hospitalizations. Patients and Methods: All patients admitted to the hospital with flu like disease and those with fever or exacerbation of any underlying disease during hospitalization without an evident cause, were prospectively enrolled. A direct immunofluorescence (DIF) of nasopharyngeal aspirate for influenza A (IA) and $B$, parainfluenza 1, 2 and 3 , adenovirus, respiratory syncytial virus (RSV) and metapneumovirus, was performed. Epidemiological and clinical data were recorded. Results: Between May and September 2012, 975 adults were admitted to the Internal Medicine Unit of Puerto Montt Hospital and in 128 (13\%) patients, DIF was carried out. DIF was positive in 44 patients (34\%) aged $65 \pm 20$ years, $68.2 \%$ females, corresponding to $4.5 \%$ of total hospitalizations. Eighty six percent of the latter had at least one co-morbidity, mainly asthma and chronic respiratory diseases in $34.1 \%$, diabetes in $29.5 \%$, cardiac problems in $25 \%$ and congestive heart failure in 20.5\%. The most common RVi were RSV $(n=21$, $48 \%)$ and IA $(n=17,39 \%)$. Six patients had a nosocomial RVi. Patients infected with IA had a significantly higher frequency of fever and bronchial hyper reactivity than those infected with RSV. RVi were associated with exacerbation of underlying disease in $62 \%$ of cases and pneumonia in $27 \%$. Two patients had a viral pericarditis. Conclusions: RVi are an important cause of adult morbidity and their detection should be routine in adult patients hospitalized during winter.

(Rev Med Chile 2014; 142: 696-701)

Key words: Influenza, Human; Pneumonia, Respiratory Tract Infections; Respiratory Syncytial Virus Infections; Viral.
L a infección por virus respiratorios (VR) es la causa más frecuente de consulta médica en el mundo ${ }^{1}$ y puede asociarse con manifestaciones diversas, desde cuadros menores y autolimitados de la vía aérea superior hasta formas graves que comprometen la vía aérea inferior como la neumonía adquirida en la comunidad (NAC). El rol patogénico de los VR es claro en la neumonía de la población pediátrica, especialmente en los lactantes, en los que el estudio etiológico contempla siempre la detección de estos agentes, principalmente mediante la técnica de inmunofluorescencia directa (IFD). En los adultos, en cambio, la importancia que se concede a los VR es menor y su búsqueda rutinaria como agentes etiológicos de NAC es mucho menos frecuente ${ }^{2,3}$. A pesar que 
los VR no son relevantes como causa de NAC en los adultos sanos, salvo en períodos epidémicos de influenza A (IA), su importancia aumenta en los adultos mayores y especialmente en aquellos con comorbilidades (CM), donde pueden ocasionar descompensaciones de enfermedades crónicas, coinfección de neumonías bacterianas o neumonía viral primaria ${ }^{4}$. Esto ha sido demostrado gracias a la introducción de nuevos métodos diagnósticos que han mejorado nuestra capacidad para identificar múltiples virus, particularmente en la NAC del adulto donde se han reconocido como potenciales agentes etiológicos hasta en 35\% de los casos, predominando IA y el virus sincicial respiratorio (VRS) ${ }^{2,3,5,6}$.

La infección por virus IA estacional y por VRS son causas predecibles de enfermedad respiratoria en invierno pero la neumonía viral primaria por IA es poco común en huéspedes inmunocompetentes en ausencia de brote epidémico ${ }^{4}$. En ancianos vacunados contra la IA en los que se documentó infección por IA sólo $5 \%$ desarrolló neumonía ${ }^{7}$. En los adultos, aquellos con enfermedad cardiopulmonar y/o estado de inmunosupresión tienen mayor riesgo de presentar enfermedad grave por VRS $^{8}$.

En el presente trabajo buscamos dimensionar la frecuencia de infección por VR en pacientes adultos hospitalizados durante los meses de invierno en un servicio de Medicina Interna del sur de Chile para caracterizar su expresión clínica y su asociación con NAC o como agentes descompensantes de las comorbilidades.

\section{Pacientes y Método}

Entre el 15 de mayo y el 15 de septiembre de 2012 se recogieron prospectivamente los datos epidemiológicos, clínicos, de laboratorio y radiológicos al ingreso de todos aquellos pacientes hospitalizados en el Servicio de Medicina del hospital de Puerto Montt por una enfermedad tipo influenza (ETI), síndrome febril o descompensación de CM sin causa evidente; además se incluyeron pacientes que estando hospitalizados por otra causa presentaron cuadros similares. Se usó la definición de ETI del Ministerio de Salud, esto es: temperatura axilar $\geq 38,5^{\circ} \mathrm{C}$, tos y otros síntomas como mialgias, odinofagia o cefalea. Los casos eran pesquisados por el médico residente y analizados diariamente en la entrega de turno matinal. A todos los pacientes se les realizó panel viral por técnica de IFD en muestra de hisopado nasofaríngeo obtenido por una enfermera de turno, buscando virus IA e influenza B (IB), parainfluenza (PI) 1, 2 y 3 , adenovirus, VRS y metapneumovirus (MPV). Se realizó la técnica de reacción en cadena de la polimerasa (PCR) de hisopado nasofaríngeo para IA e IB en aquellos casos con IFD negativa con cuadro compatible con ETI asociado a dificultad respiratoria. El diagnóstico de NAC requirió la presencia de opacidades nuevas en la radiografía de tórax con síntomas de infección del tracto respiratorio inferior y en ausencia de un diagnóstico alternativo en el seguimiento. Se consideró que la neumonía era la causa de la hospitalización en ausencia de otra razón evidente, por ejemplo una comorbilidad descompensada. En los casos con NAC se hizo una valoración de la gravedad al ingreso aplicando el modelo predictivo de Fine y cols., que considera la edad, sexo, comorbilidad, signos vitales, exámenes de laboratorio y alteraciones en la radiografía de tórax en la admisión ${ }^{9}$, y el CURB-65 (por siglas en inglés de confusión, uremia, frecuencia respiratoria, presión arterial y edad $)^{10}$ y se evaluó si cumplían con los criterios IDSA/ATS para admisión a la unidad de cuidados intensivos ${ }^{11}$.

Se consideró que existía hiperreactividad bronquial (HRB) inducida por un virus si clínicamente había obstrucción bronquial en pacientes sin antecedentes de patología obstructiva y/o de haber usado broncodilatadores inhalados. Se compararon las características clínicas de los pacientes con infección por VRS e IA, buscando identificar aspectos clínicos que permitieran diferenciarlos. Definimos que una comorbilidad descompensada era la causa de la hospitalización si así era consignado al ingreso o según la convicción clínica del equipo investigador. El diagnóstico de pericarditis requirió electrocardiograma y ecocardiografía compatible además de la opinión de un cardiólogo.

\section{Estadística}

Las variables obtenidas fueron almacenadas y analizadas usando el paquete estadístico SPSS 21 para Windows. Los resultados se expresan como valores promedio con desviación estándar. Las variables continuas fueron comparadas mediante la prueba t de Student y se empleó el test de $\chi^{2}$ o 
test exacto de Fisher cuando fue apropiado para las variables categóricas. Un valor bilateral de $\mathrm{p}<0,05$ se consideró significativo.

\section{Resultados}

En el período del estudio ingresaron al Servicio de Medicina 975 pacientes y se le tomó IFD a 128 (13\%). Se demostró infección por VR en $34 \%$ (44/128) lo que corresponde a 4,5\% de los hospitalizados (44/975). El VR más frecuente fue VRS $(\mathrm{n}=21,5 \%)$ seguido de IA $(\mathrm{n}=17,4 \%)$. Hubo 3 casos de PI 3, 2 casos con MPV y un caso de IB. En 6 casos se consideró que la infección había sido adquirida dentro del hospital y correspondieron a 3 pacientes con IA y 3 pacientes con VRS. Los pacientes con infección por VR eran en $68 \% \mathrm{mu}-$ jeres, edad media 64,7 \pm 19,8 años (24-98), 59\% eran adultos $\geq 65$ años y tuvieron una de estadía hospitalaria promedio de12,6 \pm 8,8 (2-40) días.

El 86\% (38/44) tenía una o más comorbilidades y estas fueron asma y EPOC en $34 \%$ (15/44), diabetes (DM) en $30 \%(13 / 44)$, cardiopatía en $25 \%$ (11/44), insuficiencia cardiaca congestiva (ICC) en $21 \%(9 / 44)$, bronquiectasias en $14 \%(6 / 44)$, insuficiencia renal crónica en 9\% (4/44), enfermedad crónica del hígado en $7 \%$ (3/44), cáncer y enfermedades neurológicas crónicas $5 \%$ cada una $(2 / 44)$. Hubo dos casos con lupus eritematoso sistémico, uno con insuficiencia renal crónica en peritoneodiálisis y post pulso de metilprednisolona y otro con anemia hemolítica y tratamiento inmunosupresor. Otras comorbilidades (un caso cada uno) fueron: alcoholismo, infección por VIH, vasculitis con síndrome de Sjögren y uno de inmunodeficiencia de IgA.

Las principales manifestaciones clínicas y radiológicas se describen en la Tabla 1 . Se consideró que había neumonía en $27 \%$ de los casos. La radiografía de tórax fue normal en 29\% (6/21) de los casos infectados con VRS y en $18 \%(3 / 17)$ de los con IA y fue compatible con neumonía en $19 \%(4 / 21)$ de los pacientes con VRS y en $35 \%$ (6/17) de los con IA. También hubo alteraciones radiológicas asociadas a linfagioleiomiomatosis, neumonectomía y TBC activa, un caso de cada una. No se describieron nódulos ni cavitaciones.

El 39\% (17/44) de los pacientes había consultado previo a la hospitalización una o más veces, principalmente en el servicio de atención primaria de urgencia (SAPU) $21 \%(9 / 44)$ y en el servicio de urgencia 11\% (5/44). El 18\% (8/44) de los pacientes había usado antibióticos antes de hospitalizarse (macrólidos 3, cefalosporinas 3 y amoxicilina 2). Sólo un caso estaba recibiendo oseltamivir al ingreso. El resto no había recibido medicamentos antivirales.

Aquellas condiciones que se consideraron como directamente secundarias a la acción del virus están descritas en la Tabla 2. En 27 casos

\section{Tabla 1. Cuadro clínico y exámenes de laboratorio de 44 pacientes adultos con infección por virus respiratorios}

\begin{tabular}{|c|c|c|}
\hline Síntomas & n casos & $\%$ \\
\hline Tos & 40 & 91 \\
\hline Disnea & 34 & 77 \\
\hline Fiebre & 20 & 46 \\
\hline Mialgias & 13 & 30 \\
\hline Coriza & 11 & 25 \\
\hline Escalofríos & 10 & 23 \\
\hline Cefalea & 6 & 14 \\
\hline Dolor pleurítico & 9 & 21 \\
\hline Compromiso de conciencia & 5 & 11 \\
\hline Malestar abdominal & 4 & 9 \\
\hline Odinofagia & 3 & 7 \\
\hline \multicolumn{3}{|l|}{ Radiografía de tórax } \\
\hline No realizada & 1 & \\
\hline Normal & 12 & 28 \\
\hline Opacidades intersticiales & 13 & 30 \\
\hline Opacidades alveolares & 8 & 19 \\
\hline Opacidades mixtos & 4 & 9 \\
\hline Opacidades bilaterales & 15 & 35 \\
\hline $\begin{array}{l}\text { Compromiso mayor de un } \\
\text { lóbulo }\end{array}$ & 13 & 30 \\
\hline $\begin{array}{l}\text { Sospecha de congestión } \\
\text { pulmonar }\end{array}$ & 8 & 19 \\
\hline $\begin{array}{l}\text { Progresión radiológica } \geq 50 \% \\
\text { en } 48 \mathrm{~h}\end{array}$ & 5 & 12 \\
\hline Derrame pleural & 8 & 19 \\
\hline Lesiones secuelares & 6 & 14 \\
\hline Signos vitales & \multicolumn{2}{|l|}{$(\bar{x} \pm D S)$} \\
\hline Frecuencia respiratoria (resp/min) & \multicolumn{2}{|l|}{$24 \pm 8$} \\
\hline Temperatura $\left({ }^{\circ} \mathrm{C}\right)$ & \multicolumn{2}{|c|}{$36,7 \pm 0,8(36-38,8$} \\
\hline Presión arterial sistólica $(\mathrm{mmHg})$ & \multicolumn{2}{|c|}{$128 \pm 34$} \\
\hline Frecuencia cardiaca (lat/min) & \multicolumn{2}{|l|}{$96 \pm 23$} \\
\hline \multicolumn{3}{|l|}{ Exámenes de laboratorio } \\
\hline Hematocrito (\%) & \multicolumn{2}{|c|}{$36 \pm 7(20-47)$} \\
\hline Plaquetas $\left(\mathrm{x} \mathrm{mm}^{3}\right)$ & \multicolumn{2}{|c|}{$270.953 \pm 126.585$} \\
\hline Leucocitos $\left(x \mathrm{~mm}^{3}\right)$ & \multicolumn{2}{|c|}{$12.402 \pm 6.231$} \\
\hline
\end{tabular}


Virus respiratorios en adultos hospitalizados - R. Riquelme et al

Tabla 2. Complicaciones atribuibles a infección por virus respiratorios en una cohorte de pacientes adultos hospitalizados en un servicio de medicina

\begin{tabular}{|c|c|c|}
\hline & $\mathbf{n}$ & $\%$ \\
\hline $\begin{array}{l}\text { Descompensación de enfermedades } \\
\text { crónicas }\end{array}$ & 27 & 61 \\
\hline Asma y EPOC & 9 & 21 \\
\hline Influenza A & 2 & \\
\hline Respiratorio sincicial & 7 & \\
\hline Insuficiencia cardiaca congestiva & 9 & 21 \\
\hline Influenza A & 4 & \\
\hline Respiratorio sincicial & 5 & \\
\hline Diabetes & 5 & 11 \\
\hline Influenza A & 1 & \\
\hline Respiratorio sincicial & 3 & \\
\hline Metapneumovirus & 1 & \\
\hline Daño hepático crónico & 3 & 7 \\
\hline Influenza A & 1 & \\
\hline Respiratorio sincicial & 2 & \\
\hline Lupus con anemia hemolítica (IA) & 1 & 2 \\
\hline Neumonía & 12 & 27 \\
\hline Influenza A & 6 & \\
\hline Respiratorio sincicial & 4 & \\
\hline Influenza B & 1 & \\
\hline Metapneumovirus & 1 & \\
\hline Hiperreactividad bronquial por virus & 11 & 25 \\
\hline Influenza A & 8 & \\
\hline Respiratorio sincicial & 2 & \\
\hline Influenza B & 1 & \\
\hline Pericarditis viral & 2 & 4,5 \\
\hline Parainfluenza 3 & 2 & \\
\hline
\end{tabular}

$(N=44 ;$ VRS: 21, IA: 17; PI: 2; MPV: 2; IB: 1).

(61\%) una infección por VR descompensó una enfermedad previa. Cuatro pacientes cumplían los criterios recomendados por la ATS para ingresar a UCI ( 3 casos afectados por IA y 1 por VRS) pero sólo 3 ingresaron y uno lo hizo directamente. Un paciente se conectó a ventilación mecánica invasiva y ninguno a ventilación mecánica no invasiva. Los principales exámenes de laboratorio están descritos en la Tabla 1. La presión arterial de oxígeno/fracción inspirada de oxígeno (PAFI) se determinó en 17 casos y su media fue $306 \pm$ 83 (117-442) y hubo 6 pacientes con PAFI $\leq 250$.

Se tomó hemocultivos aeróbicos (HC) en 15 casos $(34 \%)$ y cultivo de esputo en 16 . Hubo 4 casos con infección asociada: Streptococcus pneu- moniae en HC, Staphylococcus aureus meticilino resistente y dos Pneumocystis jiroveci en esputo.

La causa principal de hospitalización fue la descompensación de una CM en 21/44 (48\%) seguido de la sospecha de neumonía en 19/44 (43\%). Las principales comorbilidades descompensadas que causaron la hospitalización fueron la patología respiratoria crónica (asma, EPOC, secuelas de TBC, bronquiectasias) en $23 \%(10 / 44)$ y la ICC en $8 / 44(18 \%)$.

Recibió oseltamivir 27\% (12/44) y antibióticos $86 \%(38 / 44)$. El antibiótico más usado fue monoterapia con ceftriaxona en $20 / 44(46 \%)$ o asociado con otro antibiótico en 11 casos (25\%).

Fallecieron 2 pacientes:

1) Hombre de 79 años, portador de cardiopatía e ICC, infección por VRS. Falla respiratoria y congestión pulmonar bilateral con derrame pleural. Se consideró que no había NAC y la causa de muerte fue atribuida a ICC.

2) Mujer de 83 años, IFD y PCR (+) para IA. EPOC descompensada con NAC unilateral, categoría V de Fine y categoría 3 de CURB-65, falla respiratoria que ingresó a UCI con Glasgow 9, APACHE 17, se intubó y conectó a VM durante 15 días. Falleció por insuficiencia respiratoria. La muerte fue atribuible a influenza asociada a una neumonía. No se hizo estudio etiológico de bacterias.

La edad media de los pacientes con infección por IA fue $68 \pm 20,9$ años y la de los con infección por VRS $62,6 \pm 18$ años, pero esto no fue significativo. Tampoco hubo diferencias al comparar ambos grupos en las comorbilidades, patrones radiológicos, frecuencia y gravedad de neumonía ni mortalidad. Las únicas diferencias clínicas fueron que la infección por IA se asoció con mayor frecuencia a fiebre $(67 \%$ vs $29 \%$, OR 5,5 ; IC $95 \%$ $1,33-22,73, \mathrm{p}=0,017)$ e HRB $(47 \%$ vs $9,5 \%$ OR $8,4$; IC $95 \% 1,48-48,14 \mathrm{p}=0,016)$.

\section{Discusión}

El principal resultado de nuestro trabajo es comprobar que los VR, en invierno, juegan un importante rol en la hospitalización en un servicio de medicina interna ya sea como causa de enfermedades específicas como neumonía o pericarditis o bien descompensando alguna comorbilidad. 
Los VR en adultos han cobrado progresiva importancia en la última década. Hay evidencia que muestra que la participación de los VR en la NAC en el huésped adulto inmunocompetente varía entre $18 \%$ y $36 \%{ }^{3,5,12,13}$. En nuestro hospital, en un estudio previo de etiología de la NAC en el paciente inmunocompetente que requiere hospitalización, se demostró virus en $10 \%$ de los $\operatorname{casos}^{14}$. En esta oportunidad nos interesaba conocer el impacto de los virus respiratorios en adultos, más allá de si hubiera o no neumonía, en un servicio de medicina interna y sin usar criterios de exclusión.

Si bien es cierto este trabajo tiene las limitantes metodológicas propias de haber usado sólo un panel viral obtenido por hisopado nasofaríngeo, tiene el valor de reflejar el rendimiento que se puede obtener operacionalmente en el sistema público con los medios a nuestro alcance. La búsqueda de antígenos en secreción respiratoria por inmunofluorescencia tiene una baja sensibilidad si se compara con métodos de amplificación genómica, requiere una alta carga viral para ser positiva y se ha descrito que tiene baja sensibilidad en adultos con neumonía ${ }^{6}$. En nuestra experiencia durante la pandemia de IA H1N1 de 2009 la IFD tuvo un rendimiento de $51 \%$ en nuestro medio ${ }^{15}$. Otra limitación es que no se disponía de métodos diagnósticos para otros virus respiratorios como bocavirus, rinovirus y coronavirus ${ }^{6}$. Sin embargo, creemos interesante mostrar que bajo criterios clínicos simples se logró una positividad de la IFD de $34 \%$, equivalente a $4,5 \%$ de los pacientes hospitalizados en el Servicio de Medicina Interna durante el período de invierno. Considerando las limitaciones de la IFD, esta cifra con seguridad debería aumentar considerablemente si dispusiéramos de métodos diagnósticos moleculares.

$\mathrm{Al}$ igual que lo descrito en la literatura, encontramos una mayor frecuencia de infección por IA y VRS. El virus influenza, además de la posibilidad de neumonía viral, se asocia con un aumento de los ingresos por enfermedad pulmonar y cardiovascular y menos frecuentemente complicaciones neuromusculares ${ }^{16} \mathrm{y}$ de acuerdo al diagnóstico de alta de pacientes hospitalizados, el VRS se ha asociado con $10,6 \%$ de las neumonías, $11,4 \%$ de las exacerbaciones de la EPOC, 5,4\% de las descompensaciones cardiovasculares y 7,2\% de las crisis asmáticas ${ }^{7}$. Estos datos se correlacionan adecuadamente con nuestra población donde $86,4 \%$ tenía una o más comorbilidades y estas fueron principalmente asma y EPOC, diabetes, cardiopatía e insuficiencia cardiaca congestiva, estimándose que los virus respiratorios actuaron como factor descompensante en $61 \%$ de los casos.

Se ha descrito que las manifestaciones clínicas de la infección por virus influenza y VRS son similares en adultos que se hospitalizan y no difieren en días de hospitalización, frecuencia de neumonía, necesidad de UCI o ventilación mecánica y mortalidad $^{7}$. Nuestros pacientes con infección por IA tenían significativamente más fiebre que los pacientes infectados con VRS, similar a una serie en la que se compararon 132 casos de infección con VRS y 144 IA en una cohorte de ancianos de alto riesgo y en que los pacientes con VRS tenían mayor posibilidad de tener temperatura baja, congestión nasal y estornudos ${ }^{17}$. Sin embargo, las pequeñas diferencias encontradas por nosotros, al igual que ellos, no permiten distinguir los casos de infección por IA de los casos por VRS basándose en parámetros clínicos.

En ancianos, la infección respiratoria por virus IA y VRS se asocia a mayor riesgo de muerte en cualquier grupo de edad mayor de 65 años ${ }^{18}$ y se ha estimado que $5 \%$ de los enfermos con VRS y $6 \%$ de los casos con influenza requerirán un mayor nivel de cuidados durante la hospitalización ${ }^{7}$. Nuestra casuística es pequeña pero $9 \%$ (4/44), correspondiente a 3 casos con IA y 1 caso con VRS, cumplían con los criterios de la ATS para ingresar a la UCI y los pacientes fallecidos de 79 y 83 años, asociados a VRS e IA respectivamente, son un buen reflejo de esta población de mayor riesgo.

Creemos que nuestro trabajo contribuye al conocimiento del rol de los virus como agentes asociados a hospitalización en adultos en nuestro medio. Su presencia debe sospecharse rutinariamente en el período invernal en aquellos con NAC, pero también en aquellos con fiebre o comorbilidad descompensada sin causa clara y recomendamos el estudio sistemático mediante IFD y la incorporación de RT-PCR. La identificación precoz de los VR permitiría prevenir las infecciones nosocomiales, complicación que efectivamente ocurrió en seis de nuestros pacientes y puede ayudar a la racionalización de tratamientos antibióticos que con alguna frecuencia podrían ser inapropiados o innecesarios. Deberíamos considerar, como ya ha sido planteado ${ }^{19}$, normar la incorporación de fármacos antivirales en el tratamiento empírico de la neumonía que requiere hospitalización en 
Virus respiratorios en adultos hospitalizados - R. Riquelme et al

invierno o al menos en aquellos casos graves que van a la UCI.

\section{Referencias}

1. Anzueto A, Niederman MS. Diagnosis and treatment of rhinovirus respiratory infection. Chest 2003; 123: 166472.

2. Rabagliati R, Serri M, Montecinos L, Azócar T, Ferres M. Utilidad de la reacción de polimerasa en cadena en tiempo real en el diagnóstico de infecciones por virus respiratorio sincicial en adultos. Rev Chil Infect 2007; 24 (6): 441-5.

3. Díaz A, Fuentes G, Couble C, Uribe R, Mercado G, Soza A, et al. Etiología de la neumonía adquirida en la comunidad en adultos hospitalizados en Santiago, Chile: implicancias para las guías clínicas. Rev Chil Enf Respir 2005; 21: 23-32.

4. Falsey A. Community-acquired viral pneumonia. Clin Geriatr Med 2007; 23: 535-52.

5. Luchsinger V, Ruiz M, Zunino E, Martínez MA, Machado C, Piedra P, et al. Community-acquired pneumonia in Chile: the clinical relevance in the detection of viruses and atypical bacteria. Thorax 2013; 68: 1000-6.

6. Marcos MA, Esperatti M, Torres A. Viral pneumonia. Curr OpinI nfect Dis 2009; 22: 143-7.

7. Falsey A, Hennessey P, Formica M, Cox C, Walsh E. Respiratory syncytial virus infection in elderly and high-risk adults. N Engl J Med 2005; 352: 1749-59.

8. Murata Y. Respiratory syncytial virus infection in adults. Curr Opin Pulm Med 2008; 14: 235-40.

9. Fine M, Auble T, Yealy D, Hanusa BH, Weissfeld LA, Singer DE, et al. A prediction rule to identify low-risk patients with community-acquired pneumonia. N Engl J Med 1997; 336: 243-50.

10. Lim WS, van der Eerden MM, Laing R, Boersma WG, Karalus N, Town GI, et al. Defining community acquired pneumonia severity on presentation to hospital: an international derivation and validation study. Thorax 2003; 58: 377-82
11. Mandell L, Wunderink R, Anzueto A, Bartlett, Campbell G, Dean N, et al. Infectious Diseases Society of America/American Thoracic Society Consensus Guidelines on the Management of Community-Acquired Pneumonia in Adults. Clin Infect Dis 2007; 44 (Suppl 2): 27-72.

12. de Roux A, Marcos MA, García E, Mensa J, Ewig S, Lode $\mathrm{H}$, Torres A. Viral community-acquired pneumonia in nonimmunocompromised adults. Chest 2004; 125: 1343-51.

13. Templeton KE, Scheltinga SA, vander Eeden WC, Graffelman AW, van der Broek PJ, Claas EC. Improved diagnosis of the etiology of community-acquired pneumonia with real-time polymerase chain reaction. Clin Infect Dis 2005; 41: 345-51.

14. Rioseco ML, Riquelme R, Riquelme M, Inzunza C, Oyarzún P, Aguero Y, et al. Etiología viral en la neumonía del adulto adquirida en la comunidad en un hospital del sur de Chile. Rev Med Chile 2012; 140: 984-9.

15. Riquelme R, Riquelme M, Rioseco ML, Inzunza C, Gómez Y, Contreras C, et al. Characteristics of hospitalizad patients with $2009 \mathrm{H} 1 \mathrm{~N} 1$ influenza in Chile. Eur Respir J 2010; 36: 864-9.

16. Rothberg MB, Haessler SD, Brown RB. Complications of viral influenza. Am J Med 2008; 121: 258-64.

17. Walsh E, Petersen D, Falsey A. Is clinical recognition of respiratory syncityal virus infection in hospitalized elderly and high-risk adults possible? J Infect Dis 2007; 195: 1046-51.

18. Van Asten L, van den Wijngaard C, van Pelt W, van de Kassteele J, Meijer A, van der Hoek W, et al. Mortality attributable to 9 common infections: significant effect of influenza A, respiratory syncytial virus, influenza B, norovirus, and parainfluenza in elderly persons. J Infect Dis 2012: 206: 628-39.

19. Wiemken T, Peyrani P, Bryant K, Kelley RR, Summersgill J, Arnold F, et al. Incidence of respiratory viruses in patients with community-acquired pneumonia admitted to the intensive care unit: results from the Severe Influenza Pneumonia Surveillance (SIPS) project. Eur J Clin Microbiol Infect Dis 2013; 32: 705-10. 